\title{
Evaluating the origins of the secondary bias based on the correlation of halo properties with the linear density field
}

\author{
Xiaoyu Wang ${ }^{1,2}$, Huiyuan Wang ${ }^{1,2}$, H.J. Mo ${ }^{3}$, JingJing Shi ${ }^{4}$, Yipeng Jing ${ }^{5,1}$ \\ ${ }^{1}$ Key Laboratory for Research in Galaxies and Cosmology, Department of Astronomy, University of Science and \\ Technology of China, Hefei, Anhui 230026, China; wambyybz@mail.ustc.edu.cn, whywang@ustc.edu.cn \\ 2 School of Astronomy and Space Science, University of Science and Technology of China, Hefei 230026, China \\ 3 Department of Astronomy, University of Massachusetts, Amherst MA 01003-9305, USA \\ 4 Institute for the Physics and Mathematics of the Universe (Kavli IPMU, WPI), UTIAS, Tokyo Institutes for Advanced \\ Study, University of Tokyo, Chiba, 277-8583, Japan \\ 5 Department of Astronomy, and IFSA Collaborative Innovation Center, Shanghai Jiao Tong University, Shanghai \\ 200240, China
}

September 28, 2021

\begin{abstract}
Using two sets of large $N$-body simulations, we study the origin of the correlations of halo assembly time $\left(z_{\mathrm{f}}\right)$, concentration $\left(v_{\max } / v_{200}\right)$ and spin $(\lambda)$ with the large-scale evolved density field at given halo mass, i.e. the secondary bias. We find that the secondary bias is the secondary effect of the correlations of halo properties with the linear density estimated at the same comoving scale. Using the linear density on different scales, we find two types of correlations. The internal correlation, which reflects the correlation of halo properties with the mean linear over-density $\delta_{\mathrm{L}}$ within the halo Lagrangian radius $R_{\mathrm{L}}$, is positive for both $z_{\mathrm{f}}$ and $v_{\max } / v_{200}$, and negative for $\lambda$. The external correlation, which describes the correlation of halo properties with linear overdensity at $R>R_{\mathrm{L}}$ for given $\delta_{\mathrm{L}}$, shows trends opposite to the internal correlation. Both of the external and internal correlations depend only weakly on halo mass, indicating a similar origin for halos of different masses. Our findings offer a transparent perspective on the origin of the secondary bias. The secondary bias can be largely explained by the competition of the external and internal correlations together with the correlation of the linear density field on different scales. These two types of correlations combined can establish the complex halo-mass dependence of the secondary bias observed in the simulations.
\end{abstract}

Key words. large-scale structure of Universe - dark matter - methods: N-body simulations - methods: statistical

\section{Introduction}

Numerical $N$-body simulations have revealed that the clustering of dark matter halos depends not only on halo mass (e.g. Mo \& White 1996; Sheth et al. 2001) but also on other halo properties, such as mass assembly history, and structural and dynamical properties (Gao et al. 2005; Wechsler et al. 2006; Wetzel et al. 2007; Jing et al. 2007; Bett et al. 2007; Gao \& White 2007; Li et al. 2008; Faltenbacher \& White 2010; Lacerna \& Padilla 2011; Lazeyras et al. 2017; Xu \& Zheng 2018; Salcedo et al. 2018; Mao et al. 2018; Chue et al. 2018). The dependence of the halo clustering on halo properties other than the halo mass is usually referred to as the halo assembly bias or the secondary bias. Understanding such secondary bias of the halo population is important not only for understanding the formation of dark matter halos in the cosmic density field, but also for understanding galaxy formation and evolution in dark matter halos (see e.g. Zhu et al. 2006; Yang et al. 2006; Zentner et al. 2014; Hearin et al. 2015; Wechsler \& Tinker 2018; Wang et al. 2018; Salcedo et al. 2020).

It is well known that halo properties, such as assembly time, concentration, substructure and spin, are correlated among themselves (e.g. Jing \& Suto 2002; Zhao et al. 2003; Gao et al. 2004; Allgood et al. 2006; Hahn et al. 2007; Wang et al. 2011; Mao et al. 2018). However, these properties ex- hibit complex trends in their secondary bias, sometimes in a way different from that expected from their mutual correlations. For instance, the dependence of the halo clustering on the assembly time is usually strong for low-mass halos, but weak at the massive end (see e.g. Gao et al. 2005; Jing et al. 2007; Li et al. 2008; Mao et al. 2018; Chue et al. 2018), while the secondary bias for the halo spin parameter increases with halo mass (e.g. Bett et al. 2007; Gao \& White 2007; Faltenbacher \& White 2010; Salcedo et al. 2018). Moreover, the dependence of the secondary bias on the two halo structural parameters, halo concentration and subhalo abundance, changes sign at around the characteristic mass of collapse (Wechsler et al. 2006; Gao \& White 2007; Salcedo et al. 2018).

These results imply that the secondary bias has multiple origins. This is supported by numerous investigations in the literature, most of which focused on the secondary bias in the assembly time. For example, Wang et al. (2007) found that old small halos are usually located closer to massive structures than their younger counterparts (see also Hahn et al. 2009), a phenomenon referred to as the neighbour bias by Salcedo et al. (2018). Further studies showed that the secondary bias may also be related to the nearby cosmic web of halos (Yang et al. 2017; Paranjape et al. 2018; Ramakrishnan et al. 2019). Several processes related to the 
presence of massive neighbours have been proposed. For instance, the tidal field of the massive structure can accelerate the ambient matter and truncate the mass accretion onto small halos (Wang et al. 2007; Hahn et al. 2009; Wang et al. 2011; Shi et al. 2015; Paranjape et al. 2018; Mansfield \& Kravtsov 2020). Splashback halos that have ever passed through massive host halos may be severely stripped by the tidal force of the hosts (Ludlow et al. 2009; Wang et al. 2009). These halos with close massive companions are, therefore, expected to have an early assembly time because of the stripping, and can contribute significantly to the secondary bias (Wang et al. 2009; Li et al. 2013; Mansfield \& Kravtsov 2020; Tucci et al. 2021).

In addition to the truncation and stripping processes, Wang et al. (2011) found that dense environments can also enhance mass accretion by halos (see also Fakhouri \& Ma 2009). This process is expected to yield a trend of the halo bias with the assembly time that is different from the measured secondary bias. These authors suggested that the halo-mass dependence of the secondary bias is partly caused by the competition between two categories of processes, the truncation by large-scale tidal field and the availability of material to be accreted by halos (see also Chen et al. 2020). However, exactly how these processes contribute to the secondary bias is still unclear.

Suggestions have been made that the secondary bias in the halo concentration may share a common origin with the secondary bias in the assembly time (Han et al. 2019; Chen et al. 2020), although the two properties exhibit different trends as discussed above. Several studies (Salcedo et al. 2018; Han et al. 2019; Johnson et al. 2019; Tucci et al. 2021) pointed out that the secondary bias for the spin may have a different origin than both the assembly time and concentration. The correlation and alignment of the spin amplitude and direction with the local tidal field suggest that the tidal torques may play a key role in establishing the dependence of the halo bias on the spin parameter (Hahn et al. 2007; Shi et al. 2015; Chen et al. 2016; Wang \& Kang 2018). Unfortunately, it is still unclear how the secondary bias for the concentration and spin is established and whether it is related to the halo assembly or it is produced by completely different processes.

Attempts have also been made to understand the origin of the secondary bias from the initial conditions (e.g. Wang et al. 2007; Zentner 2007; Sandvik et al. 2007; Dalal et al. 2008; Desjacques 2008; Musso \& Sheth 2012; Shi \& Sheth 2018). For example, Wang et al. (2007) found that lowmass, older halos tend to be associated with perturbations of higher mass in the initial density field (the initial mass) that are expected to collapse into halos according to the spherical collapse model. They further found that the bias relation obtained from the initial masses in $N$-body simulations actually matches the secondary bias in the assembly time. Dalal et al. (2008) used the properties of density peaks in the initial conditions to infer the halo assembly time and concentration, and found that the general trends in the secondary bias of these two halo properties can be reproduced in their model. For massive halos, they suggested that the secondary bias reflects the statistics of the random Gaussian field (see also Zentner 2007), while for low-mass halos, they reached conclusions that are similar to those in earlier studies (e.g. Wang et al. 2007, 2009). More recently using the excursion set approach, Shi \& Sheth (2018) suggested that the secondary bias possibly reflects the correlation of densities at different scales when the density at the halomass scale is fixed.

All these results provide valuable insight into the origin of the secondary bias. However, the correlations between peak properties and halo properties are ambiguous, and the excursion set approach is not able to model the effects of the tidal truncation.

Thus, the details of the secondary bias, in particular its mass dependence, remain unresolved. It is still unclear whether or not the secondary bias for low- and high-mass halos have the same origin. It is also unclear why different halo properties exhibit different trends in their secondary bias, although they are correlated. Furthermore, since the secondary bias may already be present in the initial conditions, it is important to understand how it is connected to the secondary bias observed for the halo population in the evolved density field, and whether the initial condition or later evolution plays the more important role in determining the secondary bias.

In this paper, we use both the evolved density field (at $z=0$ ) and the linear density field to study the secondary bias of three halo properties: assembly time, concentration and spin. We use various correlation analyses to disentangle different effects. The paper is organized as follows. In Section 2, we describe the simulations, dark matter halo samples, the merger tree construction, and the quantities we use for our analyses. In section 3, we study the correlation of halo properties with the evolved and linear density fields at a typical large scale where halo bias can be measured. In Section 4, we study the correlation of halo properties with linear densities at various scales and present our findings of two types of correlation that can affect the secondary bias of halos. In Section 5, we use the two types of correlation to interpret the secondary bias for different halo properties and its dependence on halo mass. Finally, we summarize and discuss our results in Section 6.

\section{Simulations and Dark Matter Halos}

\subsection{Simulations, Halos and Merger trees}

Two simulations with different mass resolutions are used in this paper. The higher resolution one is the ELUCID simulation carried out by Wang et al. (2016) using the LGADGET code, a memory-optimized version of GADGET2 (Springel 2005). This simulation has $3072^{3}$ dark matter particles, each with a mass of $3.08 \times 10^{8} h^{-1} \mathrm{M}_{\odot}$, in a periodic cubic box of 500 comoving $h^{-1} \mathrm{Mpc}$ on a side. The other simulation has $2048^{3}$ particles in a cubic box of $1 h^{-1} \mathrm{Gpc}$ on a side, with particle mass of $8.3 \times 10^{9} h^{-1} \mathrm{M}_{\odot}$. This simulation is referred to as $\mathrm{S} 1 \mathrm{k}$ in the following. The initial conditions of the two simulations are generated at redshift of 100 by using the Zel'dovich approximation (Zel'Dovich 1970). The cosmology parameters used in the simulations are both based on WMAP5 (Dunkley et al. 2009): $\Omega_{\Lambda, 0}=0.742, \Omega_{m, 0}=0.258, \Omega_{b, 0}=0.044, \mathrm{~h}$ $=\mathrm{H}_{0} / 100 \mathrm{~km} \mathrm{~s}^{-1} \mathrm{Mpc}=0.72, \sigma_{8}=0.80$, and $n_{s}=0.96$. The characteristic collapse mass, $M_{*}$, defined as the characteristic mass scale at which the RMS of the linear density field is equal to 1.686 at the present time. For the present simulations, $M_{*}=10^{12.5} h^{-1} \mathrm{M}_{\odot}$ at $z=0$. Outputs of the two simulations are made at 100 snapshots, from $z=18.4$ to $z=0$ equally spaced in the logarithm of the expansion factor. 
Dark matter halos are identified using a friends-offriends (FOF) group-finder with a linking length $b=$ 0.2 (Davis et al. 1985). We use the SUBFIND algorithm (Springel et al. 2001) to identify gravitationally bound substructures (subhalos) within each FOF halo. This in turn makes it possible to build up halo merger trees to represent the detailed assembly histories of individual halos. Each member particle of a subhalo is assigned a weight that decreases with the absolute value of its binding energy. For a subhalo 'A' in a snapshot, its descendant is identified as the subhalo that is in the subsequent snapshot and contains the largest weighted number of particles belonging to ' $\mathrm{A}$ ', and ' $\mathrm{A}$ ' is considered as the progenitor of its descendant. In each FOF halo, the most massive subhalo is referred to as the main halo, and the branch that traces the main progenitors of the main halo back in time is referred to as the main trunk of the merging tree.

Using halo merger trees, we can also identify splashback halos (e.g. Ludlow et al. 2009; Wang et al. 2009), which are the main halos at $z=0$ but have ever been accreted by other massive halos in the past. As shown in Section 3, splashback halos mainly affect the secondary bias for small halos. These halos are expected to have experienced strong non-linear processes, in contrast to other normal halos. Since they are only a small fraction of the total halo population, we do not consider them in most of our analyses.

We select three representative halo samples at $z=0$ to show our main results. The first two, selected from ELUCID, contain 392,797 halos with $11.4 \leq \log M_{\mathrm{h}} / h^{-1} \mathrm{M}_{\odot} \leq$ 11.6 (representing low-mass halos) and 49,968 halos with $12.4 \leq \log M_{\mathrm{h}} / h^{-1} \mathrm{M}_{\odot} \leq 12.6$ (representing $M_{*}$ halos), respectively. The other one contains 9,085 halos with $13.9 \leq$ $\log M_{\mathrm{h}} / h^{-1} \mathrm{M}_{\odot} \leq 14.1$ (representing massive halos) selected from the S1k simulation. The results for other halo mass bins are presented when necessary. Note that we only show results for halos that each contain more than 800 dark matter particles, corresponding to $\log \left(M_{\mathrm{h}} / h^{-1} \mathrm{M}_{\odot}\right)>11.4$ in ELUCID and $\log \left(M_{\mathrm{h}} / h^{-1} \mathrm{M}_{\odot}\right)>12.9$ in S1k. As shown below, our results using ELUCID and S1k, which have very different mass resolutions, are similar, suggesting that mass resolution does not affect our conclusions significantly.

\subsection{Halo properties and overdensities}

In this paper, we focus on the secondary bias of halo distribution using three halo properties: the assembly time, $v_{\max } / v_{200}$ and the spin. Here we list the definitions of these three properties together with the halo mass:

- Halo mass $M_{\mathrm{h}}$ : the mass contained in the spherical region of radius $r_{200}$, centered on the most bound particle of the main halo, and within which the mean mass density is equal to 200 times the critical density.

- Halo assembly time $z_{\mathrm{f}}$ : the redshift at which $M_{\mathrm{h}}$ reaches half of its final mass at $z=0$. It is determined by tracing the main trunk of the merger tree of the halo in question.

$-v_{\max } / v_{200}$ : the ratio of the peak value of its circular velocity profile to the virial velocity. Here virial velocity is defined as the circular velocity at $r_{200}$. This parameter is often used to characterize the concentration of a halo (e.g. Gao \& White 2007).

- Halo spin $\lambda$ : defined as $\lambda=|\mathbf{J}| /\left(\sqrt{2} M_{\mathrm{h}} v_{200} r_{200}\right)$, where $J$ is the angular momentum measured by using particles within a sphere of $r_{200}$.
As mentioned in the introduction, for a given halo mass, the halo bias depends significantly on the other three halo properties. To avoid ambiguity, we refer to the dependencies as the $z_{\mathrm{f}}$ bias, the $v_{\max } / v_{200}$ bias and the $\lambda$ bias, respectively. To understand their origin, we use over-densities measured on different scales from both the evolved and linear density fields. We thus need to know the positions of the proto-halos that correspond to the halos identified at $z=0$. For each FOF halo at $z=0$, the position of the proto-halo is defined as the average position of all particles that end up in the $z=0$ halo. The following is the list of the over-densities used in our analyses:

$-\Delta_{\mathrm{b}}$, the overdensity measured within a large comoving radius range, $R_{\mathrm{b}}=[10-15] h^{-1} \mathrm{Mpc}$, centered on each halo at $z=0$. It is used to infer the halo bias factor at $z=0$.

- $\delta(R)$, the linear overdensity at $z=18.4$ at a series of comoving radius $R$, centered on the position of a protohalo.

$-\delta_{\mathrm{b}}$, the linear overdensity at the comoving radius $R_{\mathrm{b}}$ at $z=18.4$, centered on the position of a proto-halo. Note that $\delta_{\mathrm{b}}$ is exactly $\delta(R)$ when $R=R_{\mathrm{b}}$.

$-\delta_{\mathrm{L}}$, the linear overdensity at $z=18.4$ within the halo Lagrangian radius, $R_{\mathrm{L}}$, centered on the position of its proto-halo. $R_{\mathrm{L}} \equiv\left(M_{\mathrm{h}} /(4 \pi / 3 \bar{\rho})\right)^{1 / 3}$, where $M_{\mathrm{h}}$ is the halo mass of the corresponding halo at $z=0$ and $\bar{\rho}$ is the mean comoving density of the universe. Note that $\delta_{\mathrm{L}}$ is exactly $\delta(R)$ when $R$ is chosen in the range of $\left[0, R_{\mathrm{L}}\right]$. $-\delta_{\mathrm{e}}$, the linear overdensity at $z=18.4$ within $[1,1.2] R_{\mathrm{L}}$ for a proto-halo. It is exactly $\delta(R)$ when $R=$ $\left[R_{\mathrm{L}}, 1.2 R_{\mathrm{L}}\right]$.

For clarity, we use $\delta$ to denote the overdensity measured at $z=18.4$ and $\Delta$ to denote the present-day overdensity. As shown in Han et al. (2019), the bias factor at the scale from 5 to $20 h^{-1} \mathrm{Mpc}$ is well consistent with the linear theory. We thus adopt $R_{\mathrm{b}}=[10,15] h^{-1} \mathrm{Mpc}$ to measure $\Delta_{\mathrm{b}}$. For any given halo sample, the halo bias is calculated as

$b_{\mathrm{h}}=\frac{\left\langle\Delta_{\mathrm{b}}\right\rangle}{\bar{\Delta}_{\mathrm{b}, \mathrm{p}}}$

where $\langle\cdots\rangle$ denotes the average over all halos in the sample, and $\bar{\Delta}_{\mathrm{b}, \mathrm{p}}$ is the mean overdensity measured at $R_{\mathrm{b}}$ centered on all particles in the density field at $z=0$.

The linear densities used in this paper are measured from the snapshot at $z=18.4$ rather than the initial condition at $z=100$. At $z=100$, the true overdensity is very small and the shot noise of particles is important, which can lead to systematic bias in the estimate of the true linear density fluctuation. On the other hand, the overdensities obtained at $z=18.4$ are in good agreement with the prediction of the linear perturbation theory (see Appendix). Finally, we note that the Lagrangian radii, $R_{\mathrm{L}}$, for the three representative halo samples are about 1.02, 2.19 and 6.94 $h^{-1} \mathrm{Mpc}$, respectively.

\section{Halo secondary bias in evolved and linear density fields}

Fig. 1 shows the secondary bias for the three halo properties, $z_{\mathrm{f}}$ (left), $v_{\max } / v_{200}$ (middle) and $\lambda$ (right). In a given narrow mass range (0.2 dex), we select two sub-samples that 

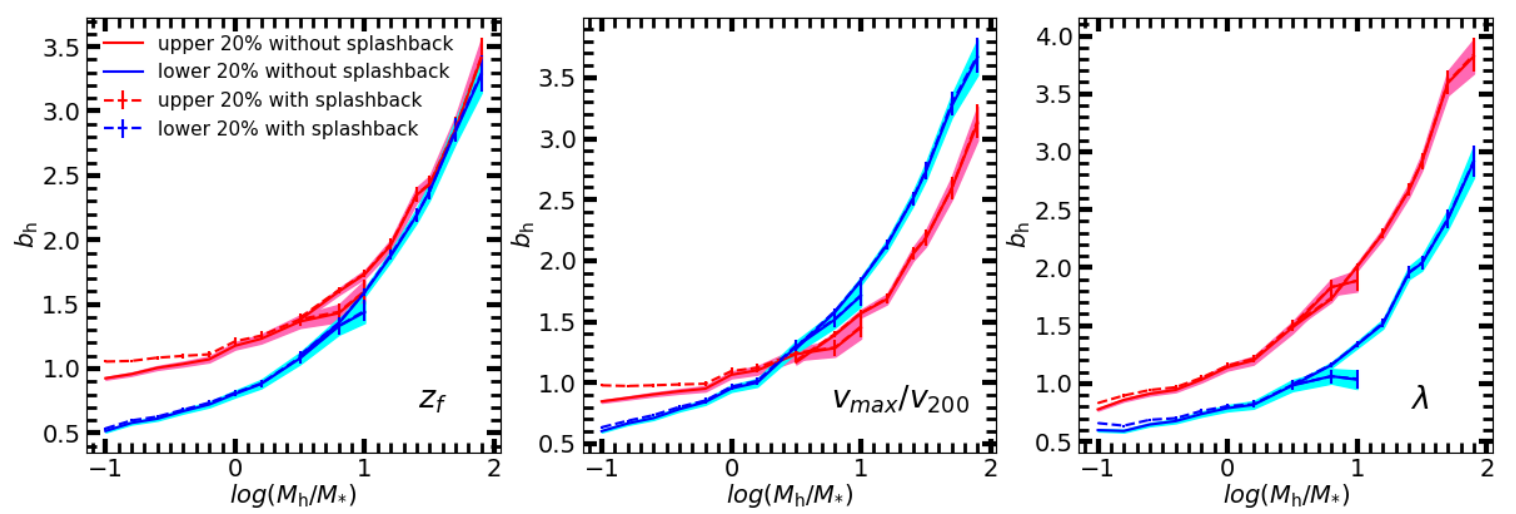

Fig. 1. Halo bias factor as a function of halo mass and other halo properties. In each panel the red (blue) lines show the results for halos in the upper (lower) $20 \%$ tails of the distribution of the halo property indicated in the panel. The dashed line shows the result for all halos, while the solid line shows the result excluding splashback halos. Results are shown for ELUCID and S1k halos in the mass ranges of $\log \left(M_{\mathrm{h}} / M_{*}\right)<1$ and $\log \left(M_{\mathrm{h}} / M_{*}\right)>0.5$, respectively. Error bars are the standard deviation calculated using 1000 bootstrap samples.
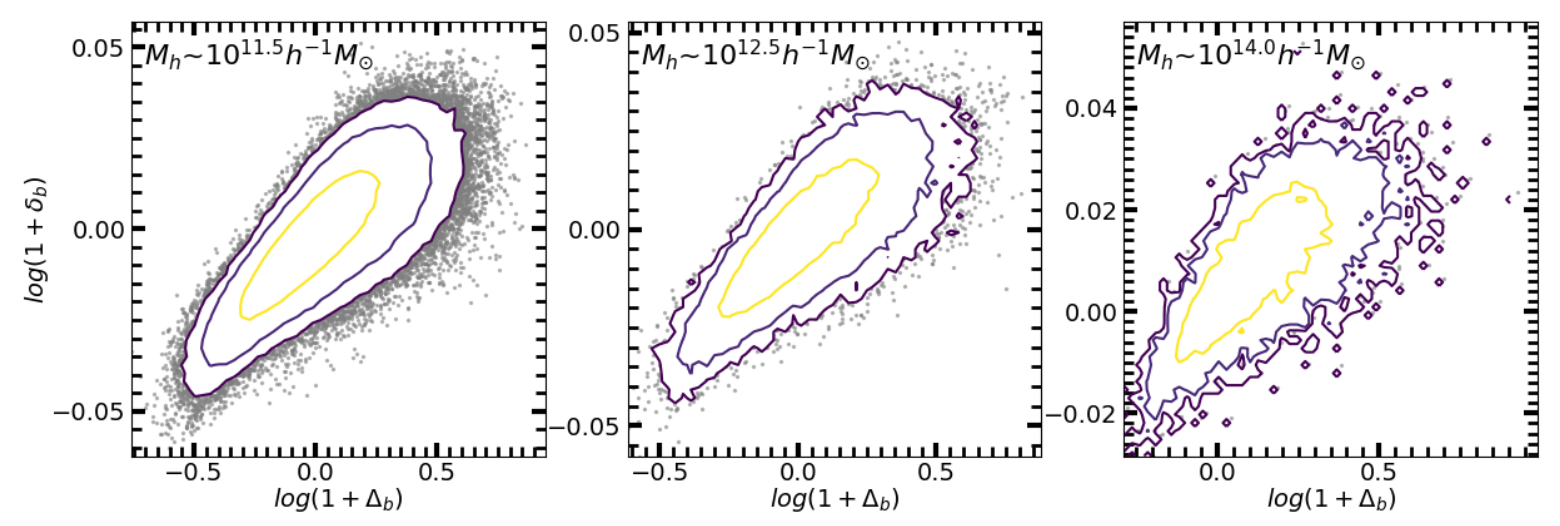

Fig. 2. The correlations between the overdensities measured at $z=18.4\left(\delta_{\mathrm{b}}\right)$ and at $z=0\left(\Delta_{\mathrm{b}}\right)$ for three representative halo samples, as indicated in the panels. Both overdensities are measured within a large comoving radius range, $R_{\mathrm{b}}=[10-15] h^{-1} \mathrm{Mpc}$. The three contour lines in each panel enclose $67 \%, 95 \%$ and $99 \%$ of halos, respectively.

consist of halos in the lower and upper 20 percent tails of the distribution of the halo property in question, respectively. We calculate the halo bias $\left(b_{\mathrm{h}}\right)$ for each of the sub-samples and show it as a function of halo mass. The dashed and solid lines show the results including and excluding splashback halos, respectively. For the ELUCID simulation, we present results for halos with $-1 \leq \log \left(M_{\mathrm{h}} / M_{*}\right) \leq 1$, while for the S1k simulation, results are shown for $\log \left(M_{\mathrm{h}} / M_{*}\right) \geq 0.5$. Although the two simulations have very different mass resolutions, their results agree with each other well in the overlapping mass range, suggesting that our results are not significantly affected by numerical resolutions.

Fig. 1 shows that splashback halos mainly affect results for low-mass halos, as expected from the fact that the fraction of the splashback population decreases with increasing halo mass (Wang et al. 2009). The exclusion of splashback halos decreases the bias for halos in the upper percentiles of the $z_{\mathrm{f}}$ and $v_{\max } / v_{200}$ distributions, while the halo bias for the lower percentiles is not affected significantly. This is expected. At low $z_{\mathrm{f}}$, halo bias depends only weakly on $z_{\mathrm{f}}$ (see e.g. Gao et al. 2005), while splashback halos on average have higher $z_{\mathrm{f}}$ (e.g. Wang et al. 2009). Different from $z_{\mathrm{f}}$ and $v_{\max } / v_{200}$, the $\lambda$ bias for the two subsamples are both significantly affected by the splashback halos. Splashback halos are expected to have experienced strong non-linear evolution, and consequently behave very differently from other halos in their relations to the linear density field. To reduce uncertainties caused by splashback halos, we exclude them in our analyses.

All the three parameters show strong secondary bias, but with very different halo-mass dependence (see also e.g. Faltenbacher \& White 2010; Salcedo et al. 2018). Older halos are usually more strongly clustered than younger ones of the same mass. However, the $z_{\mathrm{f}}$ bias becomes weaker as halo mass increases and is almost absent at $\log \left(M_{\mathrm{h}} / M_{*}\right)>$ 1 . The $v_{\max } / v_{200}$ bias is significant over the whole mass range covered. More interestingly, it changes sign around $M_{h} \sim M_{*}$, above which less concentrated halos are actually more strongly biased. The $\lambda$ bias is also strong in the whole mass range. Different from the other two parameters, its strength increases with the halo mass.

It is known that $v_{\max } / v_{200}$ increases with $z_{\mathrm{f}}$ at given halo mass (e.g. Gao et al. 2004; Han et al. 2019), and so the $z_{\mathrm{f}}$ bias and $v_{\max } / v_{200}$ bias may have a similar origin for low mass halos, as suggested by previous studies (e.g. Chen et al. 2020). However, for massive halos, the two secondary 


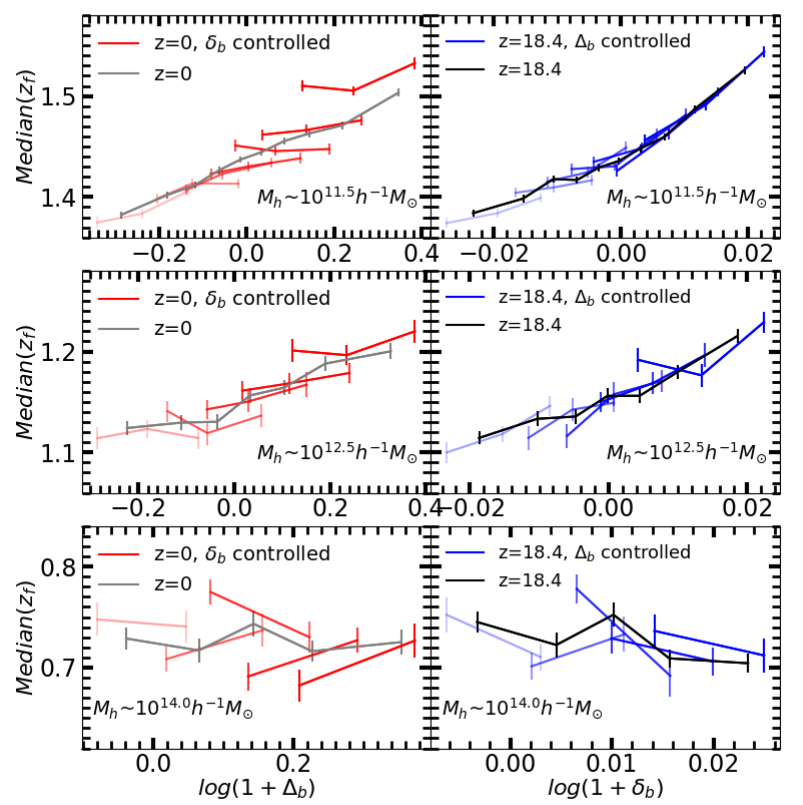

Fig. 3. The median $z_{\mathrm{f}}$ as a function of $\Delta_{\mathrm{b}}$ (left panels) and $\delta_{\mathrm{b}}$ (right panels) for three representative halo samples, as indicated in the panels. The red (blue) lines in left (right) panels show the results with $\delta_{\mathrm{b}}\left(\Delta_{\mathrm{b}}\right)$ controlled (see the text for details). The error bars show the standard deviation calculated using 1000 bootstrap samples.

biases behave differently from the expectation of the correlation between the two parameters. Moreover, it is also known that older halos tend to have smaller spin over the whole mass range (e.g. Hahn et al. 2007; Wang et al. 2011), indicating that the $z_{\mathrm{f}}$ bias and $\lambda$ bias must be caused by different processes.

To understand the origin of the secondary bias and the complex mass dependence shown above, we investigate the correlations of the halo properties with the linear density field. Here we first focus on $\delta_{\mathrm{b}}$, which is estimated at the same comoving scale as $\Delta_{\mathrm{b}}$. As shown in Fig. $2, \delta_{\mathrm{b}}$ and $\Delta_{\mathrm{b}}$ are strongly correlated, albeit with considerable variance. It is thus interesting to investigate whether their correlations with halo properties are similar. We show the median of halo properties as functions of $\Delta_{\mathrm{b}}$ and $\delta_{\mathrm{b}}$ for the three representative halo samples in Figs. 3, 4 and 5. To see which density indicator dominates the correlations, we split each of the representative halo samples into several equal-sized sub-samples according to $\Delta_{\mathrm{b}}$ or $\delta_{\mathrm{b}}$. We then show halo properties of individual subsamples of fixed $\delta_{\mathrm{b}}$ as a function of $\Delta_{\mathrm{b}}$ in the left panels (red lines), and those of fixed $\Delta_{\mathrm{b}}$ as a function of $\delta_{\mathrm{b}}$ in the right panels (blue lines). The numbers of the equal-sized subsamples are 7, 5 and 5 for the low-mass, $M_{*}$ and cluster-sized halos, respectively.

For the halo assembly time, one can see clear dependence on both $\Delta_{\mathrm{b}}$ and $\delta_{\mathrm{b}}$. The overall correlation strengths with the two densities are similar. The dependence weakens with increasing halo mass and disappears for cluster-sized halos, consistent with the results shown in Fig. 1. As one can see, for the two low mass bins, the dependence on $\Delta_{\mathrm{b}}$ becomes very weak when $\delta_{\mathrm{b}}$ is controlled. On the other

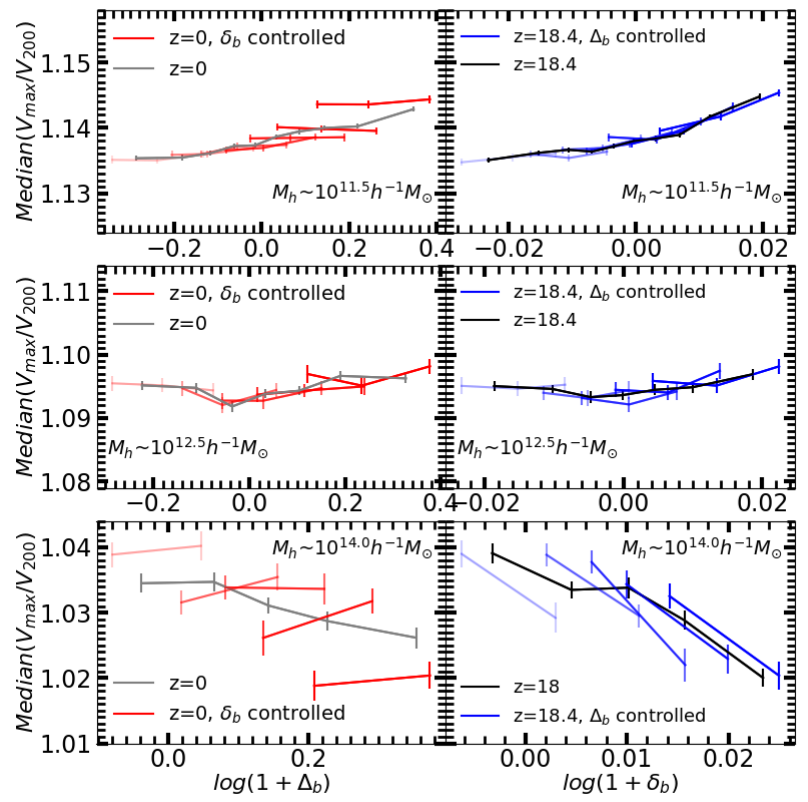

Fig. 4. The same as Fig. 3 but for $v_{\max } / v_{200}$.

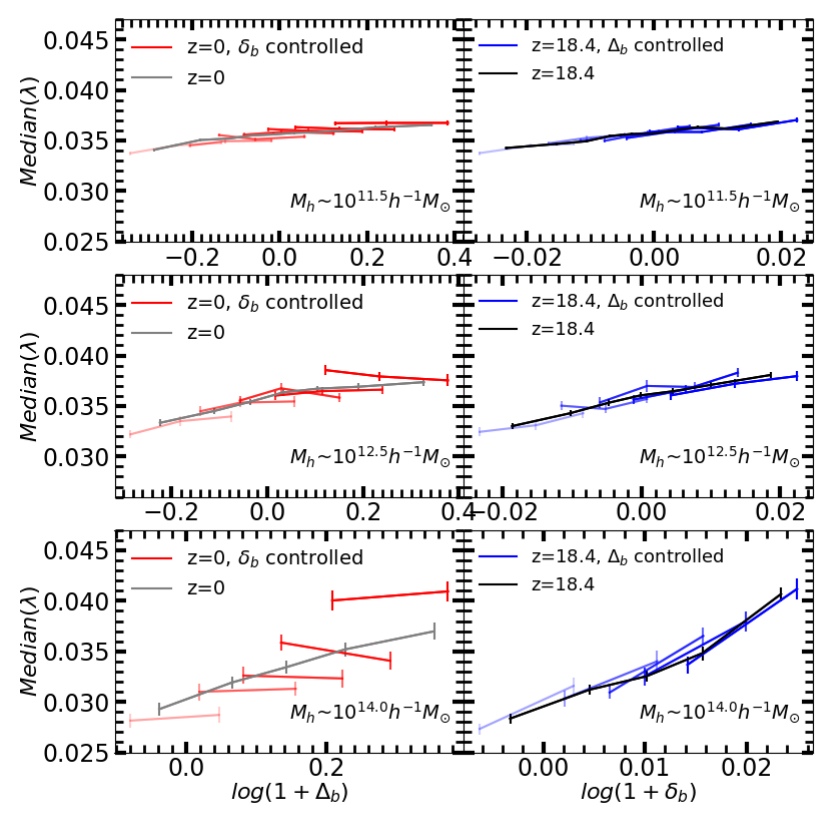

Fig. 5. Similar to Fig. 3 but for spin, $\lambda$.

hand, when $\Delta_{\mathrm{b}}$ is controlled, the correlation of $z_{\mathrm{f}}$ with $\delta_{\mathrm{b}}$ for individual sub-samples follows the overall trend closely. For the most massive halos, no clear trend can be seen.

The results for $v_{\max } / v_{200}$ are also consistent with those shown in Fig. 1. One sees that the correlation shows opposite trends for low-mass and the most massive halos. The overall trends with $\Delta_{\mathrm{b}}$ and $\delta_{\mathrm{b}}$ are similar. However, as shown in Fig. 4, for both low and high mass halos, the 


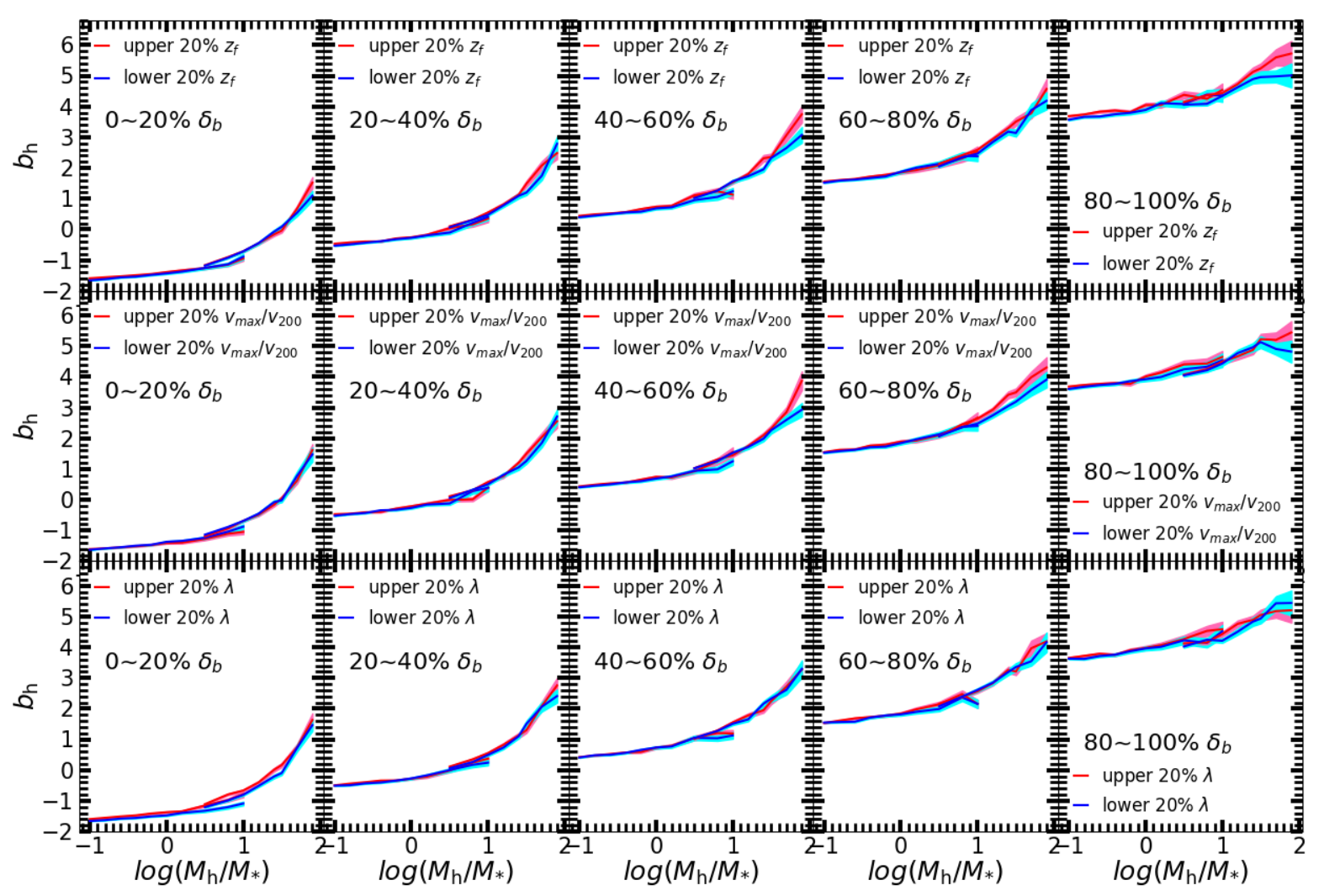

Fig. 6. Halo bias factor as a function of $M_{\mathrm{h}}, \delta_{\mathrm{b}}$ and halo properties (upper panels: $z_{\mathrm{f}}$, middle panels: $v_{\max } / v_{200}$, and lower panels: $\lambda$ ). For a given halo mass bin, we divide halos into 5 subsamples of equal size according to $\delta_{\mathrm{b}}$, as indicated in different columns. The red (blue) lines show the results for halos in the upper (lower) $20 \%$ of the distribution of a given halo property with $\delta_{\mathrm{b}}$ controlled. The symbols, color-code and shaded region are the same as in Fig. 1.

dependence on $\Delta_{\mathrm{b}}$ is absent when $\delta_{\mathrm{b}}$ is controlled, but a clear correlation (positive for low-mass halos and negative for high-mass halos) is clearly seen with $\delta_{\mathrm{b}}$ for fixed $\Delta_{\mathrm{b}}$. For halos with $M_{h} \sim M_{*}$, on the other hand, the trends of $v_{\max } / v_{200}$ with $\delta_{\mathrm{b}}$ and $\Delta_{\mathrm{b}}$ are both rather weak.

Finally, the overall correlation of $\lambda$ with the two density parameters becomes stronger as the halo mass increases, consistent with the results shown in Fig. 1. If samples are not controlled, the general trend in the $\lambda-\Delta_{\mathrm{b}}$ correlation is similar to that in the $\lambda-\delta_{\mathrm{b}}$ correlation. However, the results of the controlled samples clearly show that the linear density, $\delta_{\mathrm{b}}$, is the driving factor of the $\lambda-\Delta_{\mathrm{b}}$ correlation for all the three halo samples.

The analyses presented above demonstrate clearly that the correlations of halo properties with $\Delta_{\mathrm{b}}$ are driven by the correlations with $\delta_{\mathrm{b}}$ in all cases where significant secondary bias is detected, through the correlation between $\Delta_{\mathrm{b}}$ and $\delta_{\mathrm{b}}$. To demonstrate this in a more intuitive way, we divide halos in each mass bin into 5 subsamples of equal size according to $\delta_{\mathrm{b}}$. We then select halos in the upper and lower $20 \%$ of the distribution of a given halo property in each subsample and show the corresponding halo bias as a function of halo mass in Fig. 6. As one can see, when $\delta_{\mathrm{b}}$ is controlled, the secondary bias for all the three halo properties disappears over the entire halo mass range probed, consistent with the results shown in Fig. 3, 4 and 5. This demonstrates again that the secondary bias is more closely tied to the linear density field than to the halo properties. This also suggest that the origin of the secondary bias may be approached by investigating the correlations of halo properties with the linear density field, as we will do below.

\section{Correlations of halo properties with linear densities on various scales}

The results presented above show that halo properties are correlated with the linear over-density at a large comoving scale, $R_{\mathrm{b}}$. Wang et al. (2007) found that the halo assembly time is also correlated with the linear over-density measured within the Lagrangian radius, $\delta_{\mathrm{L}}$. These two results suggest that halo properties are correlated with the linear density field on various scales. It is thus interesting to first investigate in more detail how the correlation varies with the scale. A convenient way to characterise the correlation strength of two variables, $x$ and $y$, is to use their Pearson correlation coefficient, defined as

$\rho_{\mathrm{r}}(x, y)=\frac{\langle(x-\bar{x})(y-\bar{y})\rangle}{\sigma_{x} \sigma_{y}}$ 

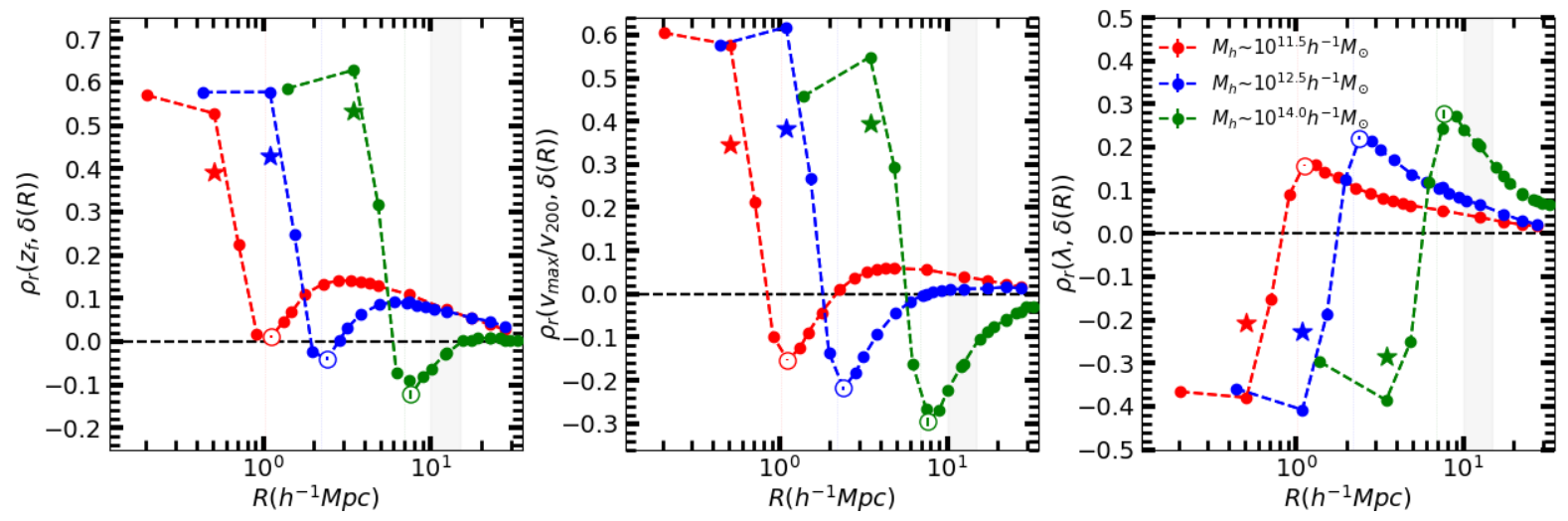

Fig. 7. Pearson correlation coefficients $\left(\rho_{\mathrm{r}}\right)$ between halo properties (left panel: $z_{\mathrm{f}}$; middle panel: $v_{\max } / v_{200}$; right panel: $\lambda$ ) and $\delta(R)$ as a function of $R$ for three representative halo samples, as indicated. The vertical error bars (usually too small to be seen) indicate the uncertainties of the coefficients, calculated using 200 bootstrap samples. The results for $\delta_{\mathrm{L}}$ and $\delta_{\mathrm{e}}$ are marked with stars and open circles, respectively. Results on other scales are shown with solid circles. The vertical dotted lines indicate $R_{\mathrm{L}}$ of the halos in question. The vertical shaded region indicates the range of $R_{\mathrm{b}}$ used to estimate the halo bias.

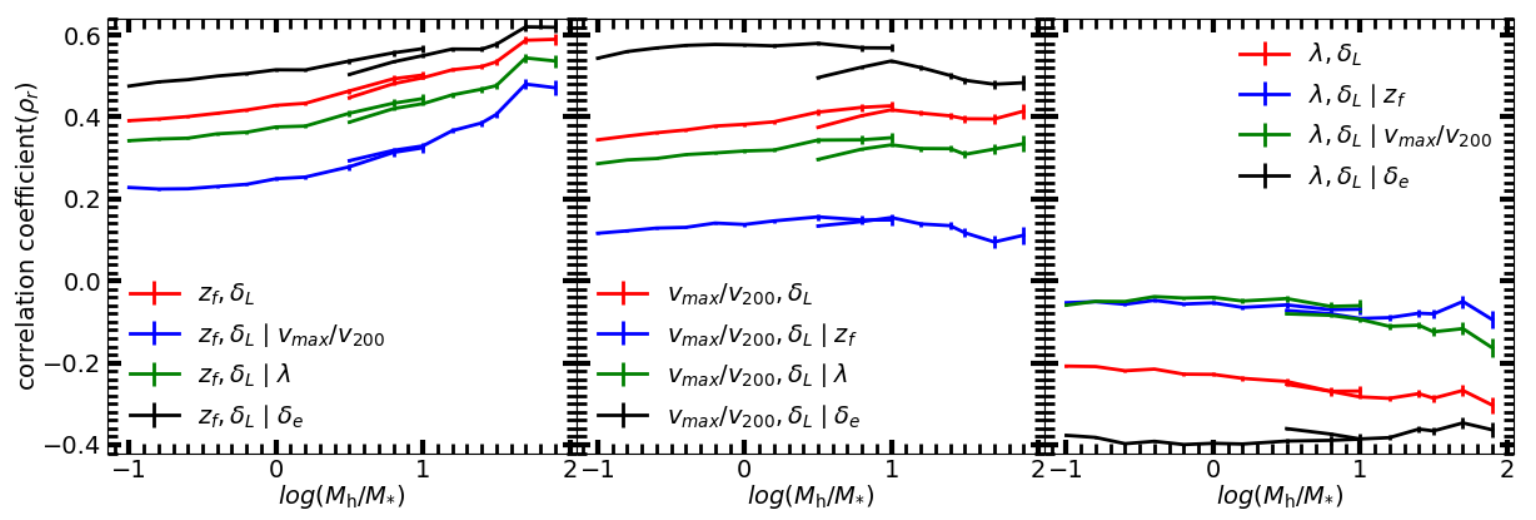

Fig. 8. Pearson coefficients for the (partial) correlations of $\delta_{\mathrm{L}}$ with halo properties, $z_{\mathrm{f}}$ (left panel), $v_{\max } / v_{200}$ (middle panel) and $\lambda$ (right panel), as a function of halo mass. As indicated in each panel, the red lines show the results of the correlations between $\delta_{\mathrm{L}}$ and halo properties, the blue and green lines show the results with another property controlled, and the black lines show the results with $\delta_{\mathrm{e}}$ controlled. The error bar shows the standard deviation calculated from 200 bootstrap samples.

where $\bar{x}(\bar{y})$ and $\sigma_{x}\left(\sigma_{y}\right)$ are the mean and standard deviation of the variable $x(y)$, respectively. As discussed in Han et al. (2019), the Pearson coefficient is a powerful tool for correlation analysis. If the two variables are roughly linearly correlated, the coefficient measures the steepness of the correlation between the corresponding normalized variables.

The Pearson coefficients, $\rho_{\mathrm{r}}$, for the correlations of halo properties with $\delta(R)$ are shown in Fig. 7. Let us first look at the coefficients at the scale $R=R_{\mathrm{b}}$ (indicated by the vertical bands), i.e. the correlations of halo properties with $\delta\left(R_{\mathrm{b}}\right)=\delta_{\mathrm{b}}$, and compare them with the results shown in the last section. The absolute values of the correlation coefficients of the three halo properties with $\delta_{\mathrm{b}}$ range from 0 to 0.2 , indicating that the secondary bias is in general a weak effect. The results show that $\delta_{\mathrm{b}}$ is positively correlated with $z_{\mathrm{f}}$ for the two low-mass samples, and that the coefficient is close to zero for the most massive halos. The coefficient for $v_{\max } / v_{200}$ changes its sign around $M_{*}$, being positive and negative for halos of lower and higher masses, respectively. The correlation coefficient for $\lambda$ is always positive and in- creases with halo mass. All of these are in good agreement with the results shown in the last section.

The scale-dependence of these correlations is complex, although there are some interesting features. In the inner region of proto-halos, i.e. $R \leq 0.6 R_{\mathrm{L}}$, the correlations are almost independent of the scale. As $R$ increases, we see a rapid change in the correlation strength and even a change in the sign of the correlations around the Lagrangian radius, $R_{\mathrm{L}}$. At $R=[1,1.2] R_{\mathrm{L}}$, the coefficients reach a (local) minimum for $z_{\mathrm{f}}$ and $v_{\max } / v_{200}$, and a maximum for $\lambda$. At $R>R_{\mathrm{L}}$, the correlations show complex dependence on halo mass, which is different from the dependence on the overdensities within proto-halos. For example, for low-mass and $M_{*}$ samples, the coefficient for $z_{\mathrm{f}}$ first increases with $R$ and then decreases gradually to zero at large scales. For cluster-sized halos, on the other hand, the scale dependence appears monotonous.

These results suggest that the overdensities inside and outside the proto-halos may affect halo properties in different ways. In the following, we therefore consider the dependence on these two types of ovserdensities separately. Specifically, we use $\delta_{\mathrm{L}}$, the linear overdensity within the 

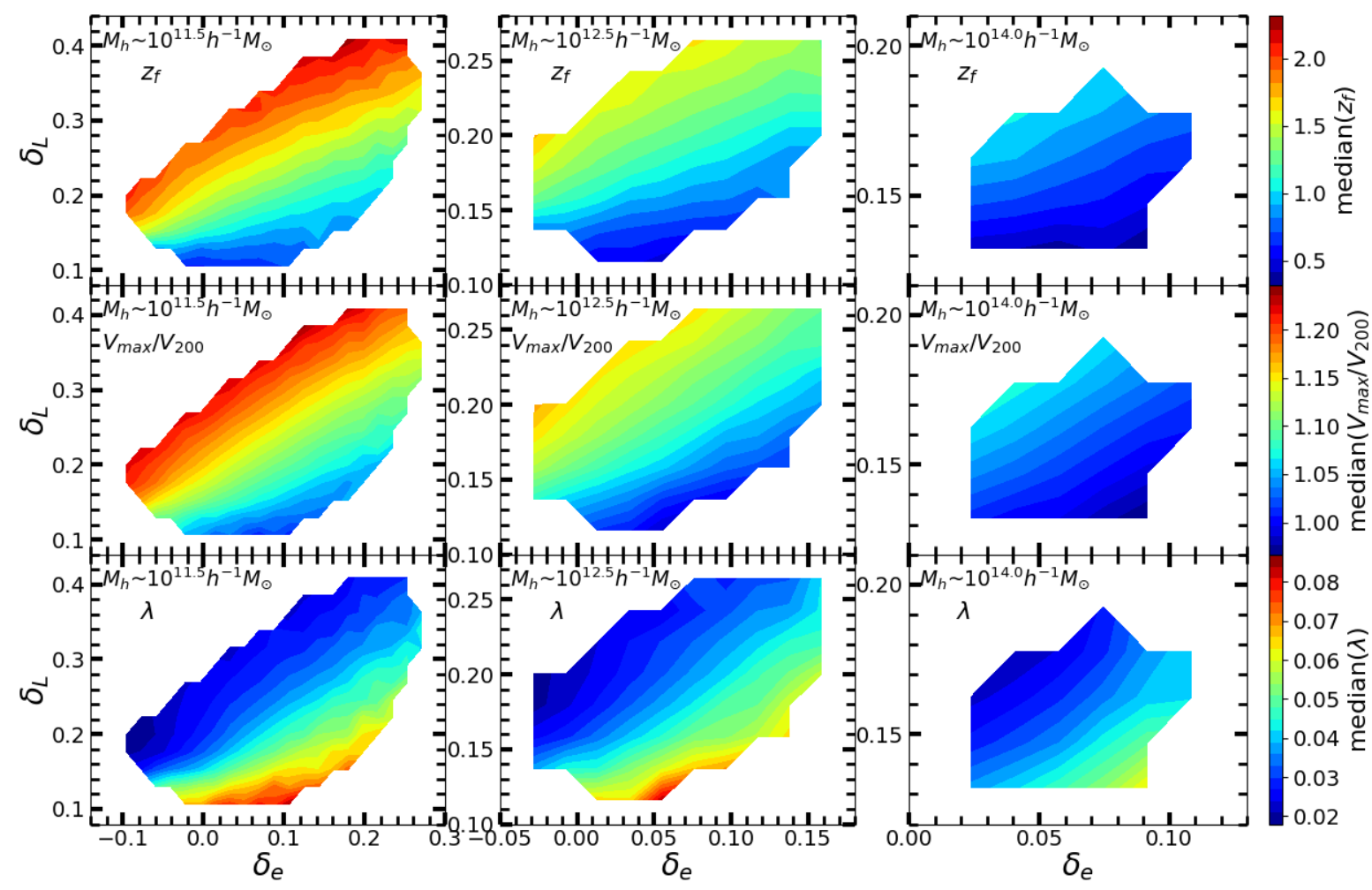

Fig. 9. Contours show median $z_{\mathrm{f}}$ (upper panels), $v_{\max } / v_{200}$ (middle panels), $\lambda$ (lower panels) as a function of $\delta_{\mathrm{e}}$ and $\delta_{\mathrm{L}}$ for three representative halo samples as indicated in the panels. Only grids containing more than 100 halos are presented.

Lagrangian radius, to represent the inside overdensity of proto-halos, and use $\delta_{\mathrm{e}}$, measured at $R=[1,1.2] R_{\mathrm{L}}$, to represent the outside overdensity (see Section 2.2 for the definition of the two quantities). We will also consider overdensities measured at larger scales when necessary.

Fig. 8 shows the correlation coefficients between the three halo properties and $\delta_{\mathrm{L}}$ as a function of halo mass. The three halo properties have different correlation strengths with $\delta_{\mathrm{L}}$, being the strongest for $z_{\mathrm{f}}\left(\rho_{\mathrm{r}}=0.4 \rightarrow 0.6\right)$ and the weakest for $\lambda\left(\rho_{\mathrm{r}}=-0.2 \rightarrow-0.3\right)$. The strong and positive correlation with the halo assembly time is well consistent with what was found in Wang et al. (2007). It is interesting that all the correlations show only a weak dependence on halo mass, which looks very different from the complex halo mass dependence of the secondary bias. We will come back to this question in the next section. Since the two simulations with very different mass resolutions give similar coefficients in the overlapping mass range, it indicates again that numerical effects are not important for the halos used in our analyses.

In Fig. 9, we show the average of the halo properties in the $\left(\delta_{\mathrm{L}}\right.$ versus $\left.\delta_{\mathrm{e}}\right)$ space. As one can see, the three halo properties exhibit strong dependence on both overdensities in all the three mass bins. Consistent with the correlation coefficient results shown in Fig. 8, both $z_{\mathrm{f}}$ and $v_{\max } / v_{200}$ have a strong positive correlation with $\delta_{\mathrm{L}}$, while $\lambda$ has a negative correlation with $\delta_{\mathrm{L}}$. We also see a clear negative correlation of both $z_{\mathrm{f}}$ and $v_{\max } / v_{200}$, and a clear positive correlation of $\lambda$, with $\delta_{\mathrm{e}}$ for given $\delta_{\mathrm{L}}$. These correlations are not always consistent with the results shown in Fig. 7 . The discrepancy is produced by the fact that $\delta_{\mathrm{L}}$ and $\delta_{\mathrm{e}}$ are correlated, so that the correlation with $\delta_{\mathrm{e}}$ depends on whether or not $\delta_{\mathrm{L}}$ is controlled. Fig. 10 shows the correlation coefficient between $\delta_{\mathrm{L}}$ and $\delta(R)$ as a function of $R$. Clearly, $\delta_{\mathrm{L}}$ is correlated with the over-density on different scales, including $\delta_{\mathrm{e}}$. The correlation coefficient decreases gradually with $R$, and depends only weakly on halo mass.

It is thus important to disentangle these correlations, and we use partial correlation coefficient to do so. The partial correlation coefficient measures the correlation coefficient between two quantities, $x$ and $y$, with a third parameter, $z$, controlled, and is defined as

$\rho_{\mathrm{r}}(x, y \mid z)=\frac{\rho_{\mathrm{r}}(x, y)-\rho_{\mathrm{r}}(x, z) \rho_{\mathrm{r}}(y, z)}{\sqrt{1-\rho_{\mathrm{r}}^{2}(x, z)} \sqrt{1-\rho_{\mathrm{r}}^{2}(y, z)}}$

(see John et al. 1989). Higher-order partial coefficient can be computed from the lower-order coefficient. For example, when two variables, $z$ and $w$, are controlled, the coefficient can be written as,

$\rho_{\mathrm{r}}(x, y \mid z, w)=\frac{\rho_{\mathrm{r}}(x, y \mid z)-\rho_{\mathrm{r}}(x, w \mid z) \rho_{\mathrm{r}}(y, w \mid z)}{\sqrt{1-\rho_{\mathrm{r}}^{2}(x, w \mid z)} \sqrt{1-\rho_{\mathrm{r}}^{2}(y, w \mid z)}}$.

The correlation coefficients between the three halo properties and $\delta_{\mathrm{L}}$, with $\delta_{\mathrm{e}}$ controlled, are plotted in Fig. 8. With $\delta_{\mathrm{e}}$ controlled, the correlations with $\delta_{\mathrm{L}}$ become significantly 


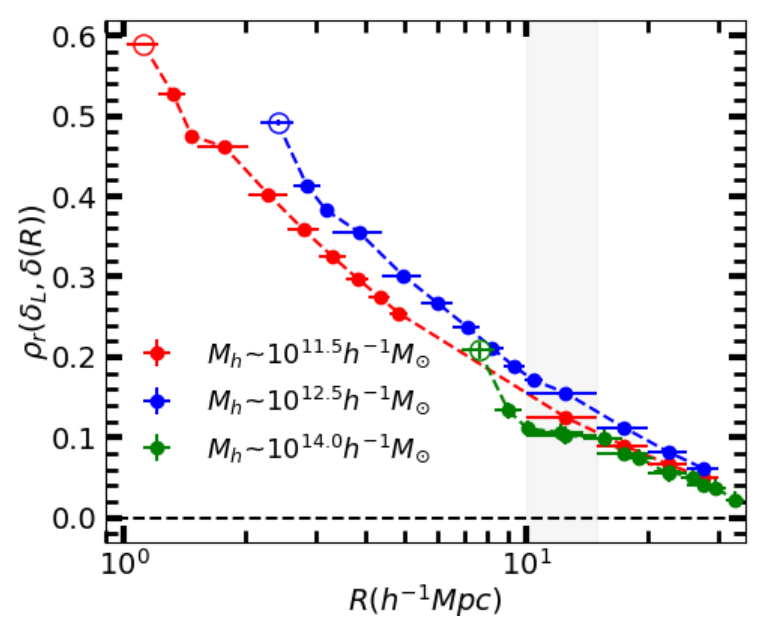

Fig. 10. Pearson coefficients between $\delta_{\mathrm{L}}$ and $\delta(R)$ as a function of $R$ for three representative halo samples. The symbols, colorcode, error bars and shaded region are the same as Fig. 7 .

stronger. This is expected, as the two densities are positively correlated (Fig. 10) but they have opposite correlations with the halo properties (Fig. 9). Note again that the halo mass dependence in these correlations are all weak.

The correlation coefficients between the halo properties and $\delta(R)$, with $\delta_{\mathrm{L}}$ controlled, are shown in Fig. 11. Here results are shown as a function of $R / R_{\mathrm{L}}$, instead of $R$. Controlling $\delta_{\mathrm{L}}$ boosts the correlation strengths between $\delta_{\mathrm{e}}$ and the halo properties, and makes the coefficients monotonous functions of $R / R_{\mathrm{L}}$ at $R / R_{\mathrm{L}}>1$. Among the three halo properties, $v_{\max } / v_{200}$ has the strongest (anti-) correlation with $\delta_{\mathrm{e}}$, and $z_{\mathrm{f}}$ the weakest. Moreover, the positive correlation of $\lambda$ with $\delta(R)$ extends to $R>5 R_{\mathrm{L}}$. In general, the correlations with $\delta_{\mathrm{e}}$ and density at larger scales are the opposite to the correlations with $\delta_{\mathrm{L}}$, consistent with the results shown in Fig. 9. Remarkably, the correlations with $\delta(R)$ at $R>R_{\mathrm{L}}$ depend on halo mass much weaker than seen in Fig. 7. To demonstrate this more clearly, we also show the results for other 12 mass bins, ranging from $10^{11.6}$ to $10^{14.4} h^{-1} \mathrm{M}_{\odot}$ and with a bin width of $0.2 \mathrm{dex}$, as the gray lines. As one can see, the controlled correlations between halo properties and the over-density at $R>R_{\mathrm{L}}$ indeed have a weak dependence on halo mass.

These results clearly suggest that two distinct types of processes may regulate the halo properties simultaneously. The first is related to the mean over-density within the proto-halos, i.e. $\delta_{\mathrm{L}}$. According to the spherical collapse model, the mean linear over-density should be the same for all halos identified at the same time. The fact that $\delta_{\mathrm{L}}$ is different for different halos indicates that the spherical collapse model is not accurate. As shown in Wang et al. (2007), some halos need a higher over-density to form, because local tidal fields may act to prevent the collapse of the outer parts of proto-halos. This may explain the positive correlations of $\delta_{\mathrm{L}}$ with $z_{\mathrm{f}}$ and $v_{\max } / v_{200}$, and the negative correlation with $\lambda$. The second is the negative correlation of the overdensity at $R>R_{\mathrm{L}}$ with both $z_{\mathrm{f}}$ and $v_{\max } / v_{200}$, and the positive correlation with $\lambda$, when $\delta_{\mathrm{L}}$ is controlled. As we will see later, these correlations are partly produced by the anti-correlation between the inner density of a proto-halo and the density exterior to $R_{\mathrm{L}}$. Both types of correlations have only weak dependence on halo mass, presumably because the linear density field is roughly scale-free over the scales concerned here. For convenience, we use internal correlations to refer to those related to $\delta_{\mathrm{L}}$, and external correlations to those related to $\delta_{\mathrm{e}}$ and over-densities on larger scales.

Since the three halo properties are correlated with each other, it is important to check whether the internal and external correlations for a specific halo property are induced by the correlations for another halo property. In Fig. 8, we show the correlation coefficients between $\delta_{\mathrm{L}}$ and one halo property with another property controlled. When $z_{\mathrm{f}}$ is fixed, the correlations with both $v_{\max } / v_{200}$ and $\lambda$ are largely reduced and close to zero. In contrast, the correlation with $z_{\mathrm{f}}$ remains strong even when $v_{\max } / v_{200}$ or $\lambda$ is fixed. This suggests that the $z_{\mathrm{f}}-\delta_{\mathrm{L}}$ correlation is the dominant one, while the other two correlations are largely the secondary effects of the $z_{\mathrm{f}}-\delta_{\mathrm{L}}$ correlation.

In Fig. 11, we show partial external correlation coefficients as functions of $R$, with $z_{\mathrm{f}}$ or $v_{\max } / v_{200}$ or $\lambda$ controlled in addition to $\delta_{\mathrm{L}}$. We see that the external correlation for $v_{\max } / v_{200}$ changes little when $z_{\mathrm{f}}$ or $\lambda$ is controlled. Similarly, $v_{\max } / v_{200}$ and $z_{\mathrm{f}}$ have only little influence on the external correlation for $\lambda$. However, the external correlation for $z_{\mathrm{f}}$ is strongly affected by the other two properties, in particular $v_{\max } / v_{200}$. When $v_{\max } / v_{200}$ is controlled, the external correlation efficiency of $z_{\mathrm{f}}$ becomes close to zero over a large range of scales. These results suggest that the external correlation of $z_{\mathrm{f}}$ is largely the secondary effect of the external correlation of $v_{\max } / v_{200}$, while the external correlation of $\lambda$ has a different origin from that of both $z_{\mathrm{f}}$ and $v_{\max } / v_{200}$.

To gain more understanding of the origin of the external correlations, we investigate the impact of the overdensity within $[0.4-0.6] R_{\mathrm{L}}$, denoted as $\delta\left(0.5 R_{\mathrm{L}}\right)$. The partial correlation between $\delta\left(0.5 R_{\mathrm{L}}\right)$ and $\delta(R)$ with $\delta_{\mathrm{L}}$ controlled is shown in Fig. 12. It is similar to that of the external correlations of $v_{\max } / v_{200}$ and $z_{\mathrm{f}}$. At a given $\delta_{\mathrm{L}}, \delta\left(0.5 R_{\mathrm{L}}\right)$ measures the density slope within the proto-halo. The anticorrelation between $\delta\left(0.5 R_{\mathrm{L}}\right)$ and the linear density field immediately exterior to $R_{\mathrm{L}}$ indicates that the density slope is coherent over a range of scales around $R_{\mathrm{L}}$. As suggested in Lu et al. (2006) and Dalal et al. (2008), the linear density profile of a proto-halo affects its accretion history and its final properties, such as $z_{\mathrm{f}}$ and $v_{\max } / v_{200}$, which may explain, at least partly, the external correlations of $v_{\max } / v_{200}$ and $z_{\mathrm{f}}$. The density slope may also affect $\lambda$, not only because it is correlated with the moment of inertial tensor of the proto-halo, which couples the proto-halo with the tidal field, but also because of its correlation with the halo assembly time over which the external tidal field operates to generate the angular momentum.

\section{On the origin of the secondary bias and its mass dependence}

Using the results obtained above, we attempt to understand the origin of the secondary bias and its mass dependence on. From Eq. 3, we can write the Pearson coefficient between a halo property, $p$, and $\delta_{\mathrm{b}}$ as

$\rho_{\mathrm{r}}\left(p, \delta_{\mathrm{b}}\right)=\alpha \rho_{\mathrm{r}}\left(p, \delta\left(r_{\mathrm{b}}\right) \mid \delta_{\mathrm{L}}\right)+\rho_{\mathrm{r}}\left(p, \delta_{\mathrm{L}}\right) \rho_{\mathrm{r}}\left(\delta_{\mathrm{b}}, \delta_{\mathrm{L}}\right)$

where $r_{\mathrm{b}}=R_{\mathrm{b}} / R_{\mathrm{L}}, \delta\left(r_{\mathrm{b}}\right)$ is exactly $\delta_{\mathrm{b}}$, and

$\alpha=\sqrt{\left(1-\rho_{\mathrm{r}}^{2}\left(p, \delta_{\mathrm{L}}\right)\right)\left(1-\rho_{\mathrm{r}}^{2}\left(\delta_{\mathrm{b}}, \delta_{\mathrm{L}}\right)\right)}$. 

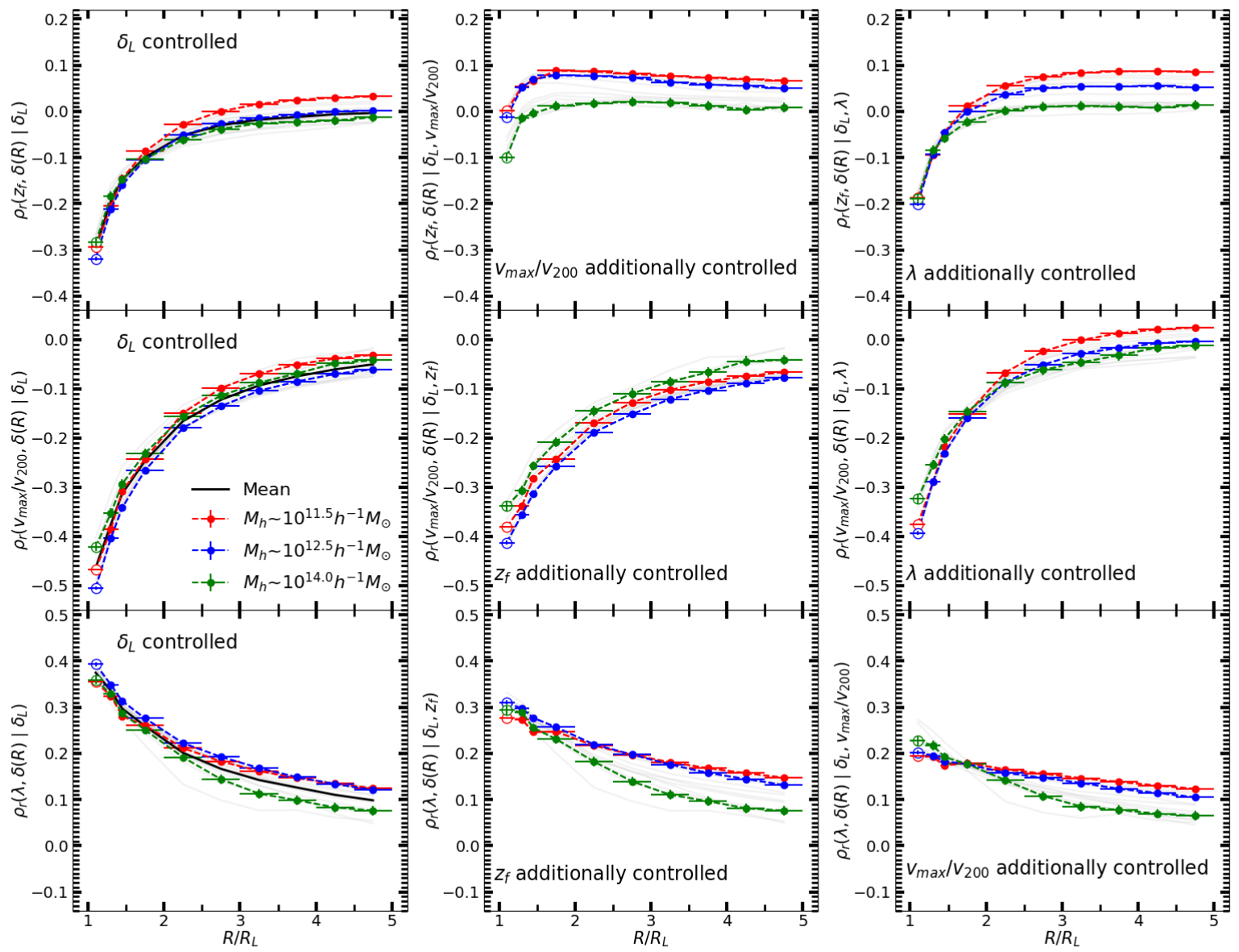

Fig. 11. Partial correlation coefficients for correlations between halo properties and $\delta(R)$, with $\delta_{\mathrm{L}}$ controlled (left panel), with $\delta_{\mathrm{L}}$ and another halo property additionally controlled (middle and right panel), as a function of $R / R_{\mathrm{L}}$. The results in left panels are computed using Eq. 3 and those in the middle and right panels using Eq. 4. The symbols, color-code and error bars are the same as Fig. 7. The gray lines show the results for other halo mass bins and the black lines show the mean results of these halo mass bins.

Since $\rho_{\mathrm{r}}\left(p, \delta_{\mathrm{L}}\right)$ ranges from $\sim-0.3$ to $\sim 0.6$ and $\rho_{\mathrm{r}}\left(\delta_{\mathrm{b}}, \delta_{\mathrm{L}}\right)$ is about 0.1 , the value of $\alpha$ ranges from 0.8 to 1 , with very weak dependence on halo mass. We thus can treat $\alpha$ as a constant close to unity.

Eq. 5 shows that the correlation of halo properties with $\delta_{\mathrm{b}}$ consists of two components, represented by the two terms on the right-hand side and related to the external and internal correlations, respectively. The secondary bias in the evolved density field, which is usually characterized by the $p-\Delta_{\mathrm{b}}$ correlation, may thus be understood in terms of the $p$ - $\delta_{\mathrm{b}}$ correlation, as $\delta_{\mathrm{b}}$ is tightly correlated with $\Delta_{\mathrm{b}}$ and the correlation is quite independent of halo mass (Fig. 2). The external correlation can result in a negative correlation of $\delta_{\mathrm{b}}$ with $z_{\mathrm{f}}$ and $v_{\max } / v_{200}$ and a positive correlation with $\lambda$. The internal correlation affects the secondary bias via the $\delta_{\mathrm{L}}-\delta_{\mathrm{b}}$ correlation, and can induce a positive correlation of of $z_{\mathrm{f}}$ and $v_{\max } / v_{200}$ with $\delta_{\mathrm{b}}$, and a negative correlation between $\lambda$ and $\delta_{\mathrm{b}}$. As shown in Figs. 8 and 11, both the external and internal components depend only weakly on halo mass. However, as we will show below, the two components combined can actually produce the mass dependence observed in the secondary bias.

Let us first look at the $z_{\mathrm{f}}$ bias. For small halos with $\log \left(M_{\mathrm{h}} / h^{-1} \mathrm{M}_{\odot}\right)=11.5$, the radius $R_{b}$ corresponds to $r_{\mathrm{b}} \equiv R_{\mathrm{b}} / R_{\mathrm{L}}>9.8$. At such a large scaled radius, the external correlation is very weak, with $\rho_{\mathrm{r}}\left(z_{\mathrm{f}}, \delta\left(r_{\mathrm{b}}\right) \mid \delta_{\mathrm{L}}\right)$ close to zero (Fig. 11). In this case, $\rho_{\mathrm{r}}\left(z_{\mathrm{f}}, \delta_{\mathrm{b}}\right) \simeq \rho_{\mathrm{r}}\left(z_{\mathrm{f}}, \delta_{\mathrm{L}}\right) \rho_{\mathrm{r}}\left(\delta_{\mathrm{b}}, \delta_{\mathrm{L}}\right)$, indicating that the positive correlation of $z_{\mathrm{f}}$ with $\delta_{\mathrm{b}}$ for these halos is mainly determined by the internal correlation. For $M_{*}$ halos, where $r_{\mathrm{b}}=[4.56,6.84]$, the contribution from the external correlation is still negligible, and so the dominant role is still played by the internal correlation. Moreover, since the $z_{\mathrm{f}}-\delta_{\mathrm{L}}$ and $\delta_{\mathrm{b}}-\delta_{\mathrm{L}}$ correlations for $M_{*}$ halos are similar to those for small halos (Fig. 7 and 10), the contribution of the internal correlation for the two halo mass samples are also similar. Indeed, as shown in Fig. 7, the correlation coefficients of $z_{\mathrm{f}}$ with $\delta_{\mathrm{b}}$ are very similar for the two halo samples. Finally, for massive cluster-sized ha- 


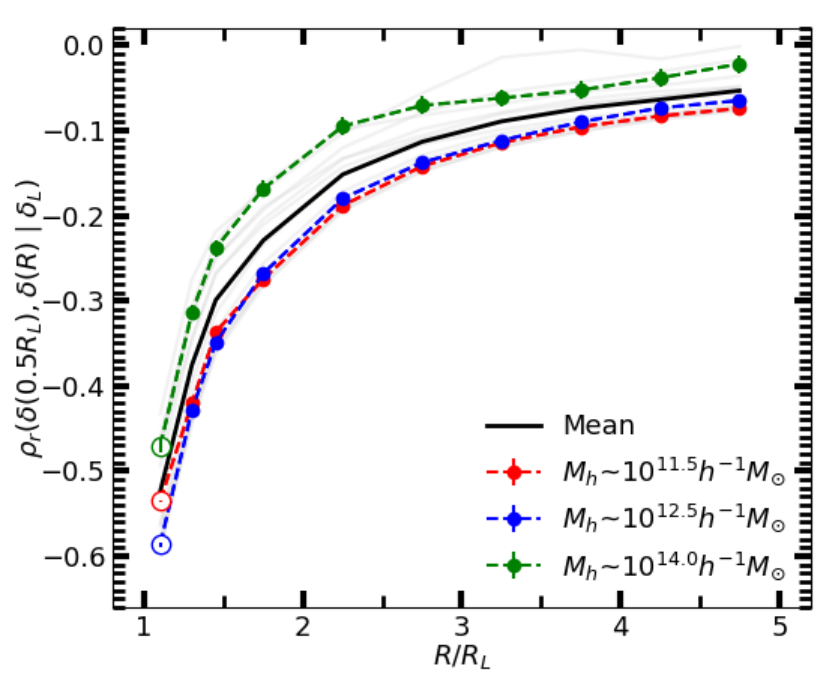

Fig. 12. Partial correlation coefficient (Eq. 3) between $\delta\left(0.5 R_{\mathrm{L}}\right)$ and $\delta(R)$, with $\delta_{\mathrm{L}}$ controlled, as a function of $R / R_{\mathrm{L}}$. Here, $\delta\left(0.5 R_{\mathrm{L}}\right)$ is the average overdensity within $[0.4-0.6] R_{\mathrm{L}}$. The symbols, color-code and error bars are the same as in Fig. 11.

los, the scaled radius $r_{\mathrm{b}}=[1.44,2.16]$. At such a scale, the external correlation becomes much stronger (Fig. 11), while the contribution of the internal correlation is only slightly stronger than that for the other two halo mass bins. Consequently, the external correlation is able to counterbalance the internal correlation, making the secondary bias for these halos very weak.

Similar analysis can be made for $v_{\max } / v_{200}$. The internal correlation of $v_{\max } / v_{200}$ is weaker, while the external correlation is stronger, than that of $z_{\mathrm{f}}$, making the external correlation more important for the $v_{\max } / v_{200}$ bias. This also makes the mass-dependence of the $v_{\max } / v_{200}$ bias different from that of the $z_{\mathrm{f}}$ bias. For small halos, the $v_{\max } / v_{200}$ bias is still dominated by the internal correlation, because their $\rho_{\mathrm{r}}\left(v_{\max } / v_{200}, \delta\left(r_{\mathrm{b}}\right) \mid \delta_{\mathrm{L}}\right)$ is close to zero (Fig. 11). Thus, a positive $v_{\max } / v_{200}-\delta_{\mathrm{b}}$ correlation is expected, consistent with the results shown in Figs. 1 and 4 . For $M_{*}$ halos, the internal and external correlations become comparable, so that $v_{\max } / v_{200}$ is independent of $\delta_{\mathrm{b}}$. For massive clustersized halos, where $r_{\mathrm{b}}$ becomes small, the external correlation becomes the dominant one, producing a negative correlation between $v_{\max } / v_{200}$ and $\delta_{\mathrm{b}}$. The change in the sign of the $v_{\max } / v_{200}$ bias is thus a result of the competition between the two correlations.

Finally for the spin, the internal correlation is even weaker, and thus plays only a minor role in producing the $\lambda$ bias. As shown in Fig. 11, the external correlation for the spin decreases with $R / R_{\mathrm{L}}$ slowly, and so the dominant effect for the $\lambda$ bias is always the external correlation over the entire mass range. Since $r_{\mathrm{b}}=R_{\mathrm{b}} / R_{\mathrm{L}}$ decreases with increasing halo mass and the external correlation is stronger at smaller $r_{\mathrm{b}}$, an increasing trend with halo mass is produced, as shown in Figs. 1, 5 and 7.

The analyses above suggest that the secondary bias observed in the evolved density field is primarily produced by the internal and external correlations of halo properties with the linear density field, and that these correlations provide a transparent way to understand the origin of the secondary bias. We note that the results are not sensitive to the choice of $R_{\mathrm{b}}$, on which the halo bias is estimated. Indeed, we reached very similar conclusions using other scales, although the details may be different. Other factors, which are not included in our analysis, may also contribute to or affect the secondary bias. For example, both the external and internal correlations show weak but complicated dependence on halo mass, indicating that the shape of the linear power spectrum may also play a role. In addition, the correlations of the halo properties with $\delta_{\mathrm{b}}$ are not expected to be exactly the same as those with $\Delta_{\text {b }}$, estimated at $z=0$, although these two overdensities are correlated. As shown in Figs. 3, 4 and 5, the non-linear evolution of the density field around halos weakens the strength of the secondary bias slightly, presumably because of the mixing of scales of the linear density field in the evolved field.

\section{Summary and discussion}

In this paper, we use two $N$-body simulations, ELUCID and S1k, to study the origin of the secondary bias for halo assembly time $\left(z_{\mathrm{f}}\right)$, concentration $\left(v_{\max } / v_{200}\right)$ and $\operatorname{spin}(\lambda)$, and its dependence on halo mass. Splashback halos, which have experienced strong non-linear processes, are excluded from our analyses. Our study is based on the correlations of these halo properties with linear densities at various scales. The main results can be summarized as follows.

- We find that the correlations of halo properties with the density measured at $R_{\mathrm{b}}=[10,15] h^{-1} \mathrm{Mpc}$ are stronger in the linear density field than in the evolved density field at $z=0$. The secondary bias in the evolved density field is the secondary effect of the correlations of halo properties with the linear density (Figs. 3, 4, 5 and 6) through the correlation of the two densities (Fig. 2).

- The correlations of halo properties with the linear density field vary rapidly and even change signs around the halo Lagrangian radius and approach zero at larger scales (Fig. 7). This suggests that the linear densities inside and outside the proto-halos affect the final halo properties in different ways.

- The three halo properties are strongly correlated with the average linear density within the Lagrangian radius, $\delta_{\mathrm{L}}$ (Fig. 8). The $z_{\mathrm{f}}$ and $v_{\max } / v_{200}$ of halos increase with $\delta_{\mathrm{L}}$, while $\lambda$ exhibits a negative correlation with $\delta_{\mathrm{L}}$. The Pearson correlation coefficient shows that all the three correlations depend only weakly on the halo mass. Our results suggest that the internal correlations of $v_{\max } / v_{200}$ and $\lambda$ are the secondary effects of the internal correlation of $z_{\mathrm{f}}$.

- At a given $\delta_{\mathrm{L}}$, all the three halo properties are correlated with $\delta\left(R / R_{\mathrm{L}}\right)$ at $R>R_{\mathrm{L}}$ in a way opposite to the internal correlation (Figs. 9 and 11). The conditional correlations, with $\delta_{\mathrm{L}}$ fixed, show only weak dependence on halo mass for all the three halo properties. The external correlations for $z_{\mathrm{f}}$ and $v_{\max } / v_{200}$ appear to share the same origin that may be partly related to the correlation of the inner slope of the proto-halos with the density field on large scales; the external correlation of $\lambda$ may have a different origin.

- The internal and external correlations are the main drivers of the secondary bias (Eq. 5). For the $z_{\mathrm{f}}$ bias, the internal correlation dominates for almost the whole range of the halo mass, and the contributions of the two 
types of correlation are comparable for cluster-sized halos. For the $v_{\max } / v_{200}$ bias, the internal correlation dominates for low-mass halos and the external correlation dominates for cluster-sized halos, while the two correlations are comparable for $M_{*}$ halos. For the $\lambda$ bias, the external correlation dominates over the whole mass range and the internal correlation only plays a minor role.

Our analysis provides a new perspective on the nature of the secondary bias. The results suggest that the combination of the internal and external correlations in the linear density field drives the secondary bias of the three halo properties considered here, and that the mass dependence of the secondary bias is the result of the competition of the two correlations and the mass dependence of the contribution of the external correlation. We therefore need to understand the mechanisms that produce the internal and external correlations, in order to fully understand the origin of the secondary bias.

The internal correlation of $z_{\mathrm{f}}$ has been studied in Wang et al. (2007), who found that the proto-halo of a $z=0$ halo with high $z_{\mathrm{f}}$ is usually located in the vicinity of massive structures. They thus suggested that the large-scale tidal field associated with (neighbour halos or/and large scale structures) the high density can truncate the accretion of the material in the outskirt of the proto-halo(see also Salcedo et al. 2018). The stronger the tidal field is, the larger the truncation effect and the higher the assembly redshift. This can explain the $z_{\mathrm{f}}-\delta_{\mathrm{L}}$ correlation. However, they suggested that this effect is only important for small halos (see also e.g. Dalal et al. 2008; Mansfield \& Kravtsov 2020), which is not consistent with our results that this effect is equally important for cluster-sized halos. This indicates that the tidal truncation may also be important for massive halos.

The external correlation can produce a secondary bias effect that is totally opposite to the internal correlation. The external correlation is complicated and more than one mechanism may be relevant. For example, previous studies (e.g. Fakhouri \& Ma 2009; Wang et al. 2011) suggested that dense environments can enhance the frequency of halo merging, and mass accretion, producing an effect similar to that of the external correlation we observe. The inner density profile within a proto-halo, which is found to correlate with large-scale density (Fig. 12), can also affect the assembly history and concentration of the halo (e.g. Lu et al. 2006; Dalal et al. 2008). A steeper initial profile, in general, produces an older and more concentrated final halo, leading to the external correlation. External tidal field can not only truncate mass accretion onto a halo but can also accelerate the tangential velocity of the material around the halo and enhance the spin (e.g. Shi et al. 2015). This may explain the significant positive correlation between the spin and linear density at large scales. The steepness of the initial density profile, which is correlated with the large-scale density field, can also affect the spin through its correlation with the moment of the inertial tensor of the proto-halo and its correlation with the assembly time over which the external tidal field operates to generate the angular momentum.

Clearly, further investigations are still needed to understand the details of these mechanisms and how they affect the properties of halos. A detailed understanding of these mechanisms is essential not only for understanding the ori- gin of the secondary bias, but also for constructing a comprehensive picture of halo formation in the cosmic density field. The results obtained here pave the way toward such a goal.

\section{Acknowledgments}

We thank the anonymous referee for a useful report that significantly improves the paper. This work is supported by the National Key R\&D Program of China (grant No. 2018YFA0404503), the National Natural Science Foundation of China (NSFC, Nos. 11733004, 11890693, and 11421303), and the Fundamental Research Funds for the Central Universities. We acknowledge the science research grants from the China Manned Space Project with NO. CMS-CSST-2021-A03. The work is also supported by the Supercomputer Center of University of Science and Technology of China.

\section{References}

Allgood, B., Flores, R. A., Primack, J. R., et al. 2006, MNRAS, 367, 1781

Bett, P., Eke, V., Frenk, C. S., et al. 2007, MNRAS, 376, 215

Chen, S., Wang, H., Mo, H. J., \& Shi, J. 2016, ApJ, 825, 49

Chen, Y., Mo, H. J., Li, C., et al. 2020, ApJ, 899, 81

Chue, C. Y. R., Dalal, N., \& White, M. 2018, JCAP, 2018, 012

Dalal, N., White, M., Bond, J. R., \& Shirokov, A. 2008, ApJ, 687, 12

Davis, M., Efstathiou, G., Frenk, C. S., \& White, S. D. M. 1985, ApJ, 292,371

Desjacques, V. 2008, MNRAS, 388, 638

Dunkley, J., Komatsu, E., Nolta, M. R., et al. 2009, ApJS, 180, 306

Fakhouri, O. \& Ma, C.-P. 2009, MNRAS, 394, 1825

Faltenbacher, A. \& White, S. D. M. 2010, ApJ, 708, 469

Gao, L., Springel, V., \& White, S. D. M. 2005, MNRAS, 363, L66

Gao, L. \& White, S. D. M. 2007, MNRAS, 377, L5

Gao, L., White, S. D. M., Jenkins, A., Stoehr, F., \& Springel, V. 2004, MNRAS, 355, 819

Hahn, O., Porciani, C., Carollo, C. M., \& Dekel, A. 2007, MNRAS, 375,489

Hahn, O., Porciani, C., Dekel, A., \& Carollo, C. M. 2009, MNRAS, 398, 1742

Han, J., Li, Y., Jing, Y., et al. 2019, MNRAS, 482, 1900

Hearin, A. P., Watson, D. F., \& van den Bosch, F. C. 2015, MNRAS, 452,1958

Jing, Y. P. \& Suto, Y. 2002, ApJ, 574, 538

Jing, Y. P., Suto, Y., \& Mo, H. J. 2007, ApJ, 657, 664

John, N., William, W., \& Whitmore, G. A. 1989

Johnson, J. W., Maller, A. H., Berlind, A. A., Sinha, M., \& HolleyBockelmann, J. K. 2019, MNRAS, 486, 1156

Lacerna, I. \& Padilla, N. 2011, MNRAS, 412, 1283

Lazeyras, T., Musso, M., \& Schmidt, F. 2017, JCAP, 2017, 059

Li, R., Gao, L., Xie, L., \& Guo, Q. 2013, MNRAS, 435, 3592

Li, Y., Mo, H. J., \& Gao, L. 2008, MNRAS, 389, 1419

Lu, Y., Mo, H. J., Katz, N., \& Weinberg, M. D. 2006, MNRAS, 368, 1931

Ludlow, A. D., Navarro, J. F., Springel, V., et al. 2009, ApJ, 692, 931

Mansfield, P. \& Kravtsov, A. V. 2020, MNRAS, 493, 4763

Mao, Y.-Y., Zentner, A. R., \& Wechsler, R. H. 2018, MNRAS, 474, 5143

Mo, H. J. \& White, S. D. M. 1996, MNRAS, 282, 347

Musso, M. \& Sheth, R. K. 2012, MNRAS, 423, L102

Paranjape, A., Hahn, O., \& Sheth, R. K. 2018, MNRAS, 476, 3631

Ramakrishnan, S., Paranjape, A., Hahn, O., \& Sheth, R. K. 2019, MNRAS, 489, 2977

Salcedo, A. N., Maller, A. H., Berlind, A. A., et al. 2018, MNRAS, 475,4411

Salcedo, A. N., Zu, Y., Zhang, Y., et al. 2020, arXiv e-prints, arXiv:2010.04176

Sandvik, H. B., Möller, O., Lee, J., \& White, S. D. M. 2007, MNRAS, 377,234

Sheth, R. K., Mo, H. J., \& Tormen, G. 2001, MNRAS, 323, 1

Shi, J. \& Sheth, R. K. 2018, MNRAS, 473, 2486

Shi, J., Wang, H., \& Mo, H. J. 2015, ApJ, 807, 37 
Springel, V. 2005, MNRAS, 364, 1105

Springel, V., White, S. D. M., Tormen, G., \& Kauffmann, G. 2001, MNRAS, 328, 726

Tucci, B., Montero-Dorta, A. D., Abramo, L. R., Sato-Polito, G., \& Artale, M. C. 2021, MNRAS, 500, 2777

Wang, H., Mo, H. J., Chen, S., et al. 2018, ApJ, 852, 31

Wang, H., Mo, H. J., \& Jing, Y. P. 2009, MNRAS, 396, 2249

Wang, H., Mo, H. J., Jing, Y. P., Yang, X., \& Wang, Y. 2011, MNRAS, 413, 1973

Wang, H., Mo, H. J., Yang, X., et al. 2016, ApJ, 831, 164

Wang, H. Y., Mo, H. J., \& Jing, Y. P. 2007, MNRAS, 375, 633

Wang, P. \& Kang, X. 2018, MNRAS, 473, 1562

Wechsler, R. H. \& Tinker, J. L. 2018, ARA\&A, 56, 435

Wechsler, R. H., Zentner, A. R., Bullock, J. S., Kravtsov, A. V., \& Allgood, B. 2006, ApJ, 652, 71

Wetzel, A. R., Cohn, J. D., White, M., Holz, D. E., \& Warren, M. S. 2007, ApJ, 656, 139

Xu, X. \& Zheng, Z. 2018, MNRAS, 479, 1579

Yang, X., Mo, H. J., \& van den Bosch, F. C. 2006, ApJ, 638, L55

Yang, X., Zhang, Y., Lu, T., et al. 2017, ApJ, 848, 60

Zel'Dovich, Y. B. 1970, A\&A, 500, 13

Zentner, A. R. 2007, International Journal of Modern Physics D, 16, 763

Zentner, A. R., Hearin, A. P., \& van den Bosch, F. C. 2014, MNRAS, 443, 3044

Zhao, D. H., Jing, Y. P., Mo, H. J., \& Börner, G. 2003, ApJ, 597, L9

Zhu, G., Zheng, Z., Lin, W. P., et al. 2006, ApJ, 639, L5

\section{Appendix A: The linear field at $z=18.4$ and its relation to the initial condition}

We use the simulation snapshots at $z=18.4$, rather than the initial condition (at $z=100$ ), to calculate the linear overdensities. Fig. A.1 shows the comparison of the overdensities within the halo Lagrangian radius measured at $z=18.4$ with the initial condition for the three representative halo samples, together with the prediction of the linear theory. Here, we use $\delta_{\mathrm{L}}$ and $\delta_{\mathrm{L}}^{\mathrm{i}}$ to denote the overdensities at $z=18.4$ and $z=100$, respectively. One can see that the median values of the three correlations are consistent with the prediction of the linear perturbation theory, indicating that the density field at $z=18.4$ is still in the linear regime. These results justify the use of the simulation data at $z=18.4$ to estimate the linear densities.

When the mass resolution is not sufficiently high, there is a systematical problem in the initial condition due to shot noise. This problem is particularly severe for halos containing small number of particles. As one can see, for $M_{*}$ and cluster-sized halos that contain at least 8,000 particles in each halo, the correlations are very tight, while for the low-mass halos, each of which has only about 800 particles, the correlation is much poorer. Thus, for halos with only about 800 particles, the overdensities measured from the initial condition are severely affected by the shot noise. To demonstrate this, we show the results for halos of $\log \left(M_{\mathrm{h}} / \mathrm{M}_{\odot}\right) \sim 13$ of both simulations in Fig. A.2. We choose this mass bin, because the $\mathrm{S} 1 \mathrm{k}$ halos contain about 800 particles, similar to the low-mass halo sample. If shot noise were not important for these S1k halos, one would expect similar correlation between the two simulations. However, the correlation is much weaker for S1k than for ELUCID. Clearly, shot noise can severely affect the measurements of the linear densities from the initial condition for halos containing less than 800 particles. We thus choose the snapshots at $z=18.4$ to calculate the linear overdensities rather than initial condition. 

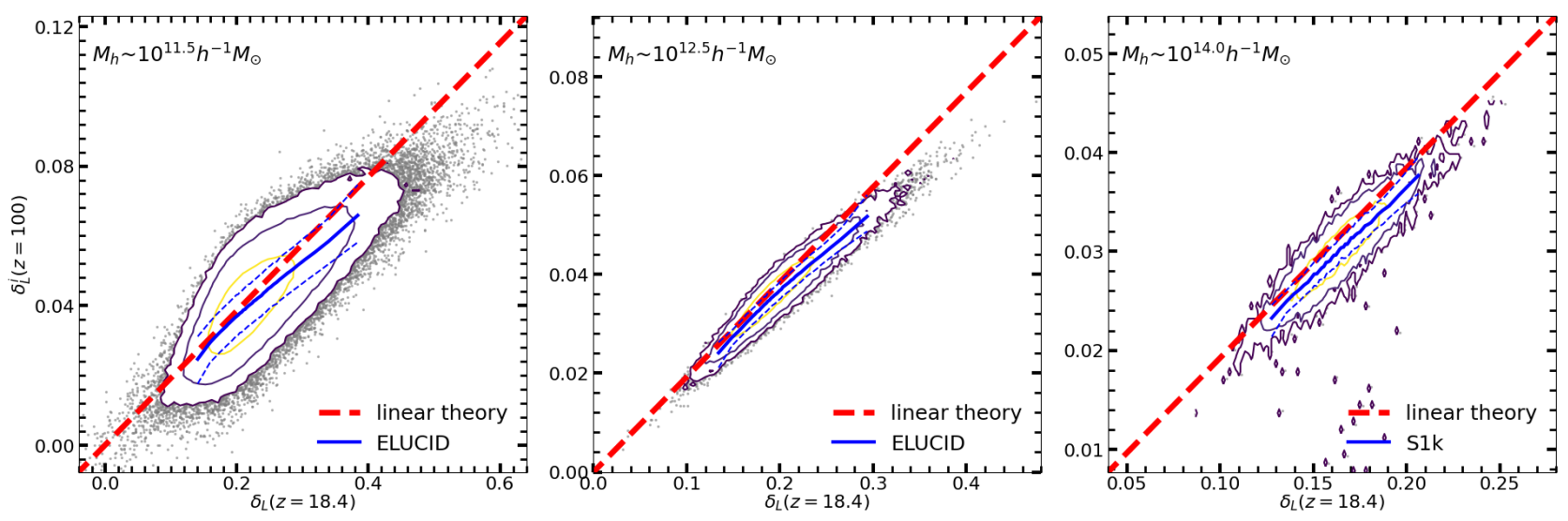

Fig. A.1. Contours show the correlation between $\delta_{\mathrm{L}}^{i}$ and $\delta_{\mathrm{L}}$ for three representative halo samples. The three contour lines in each panel enclose $67 \%, 95 \%$ and $99 \%$ of halos. The blue solid line shows the median value and the blue dash lines show $1 \sigma$ dispersion around the median relation. The red dash lines show the prediction of the linear theory.
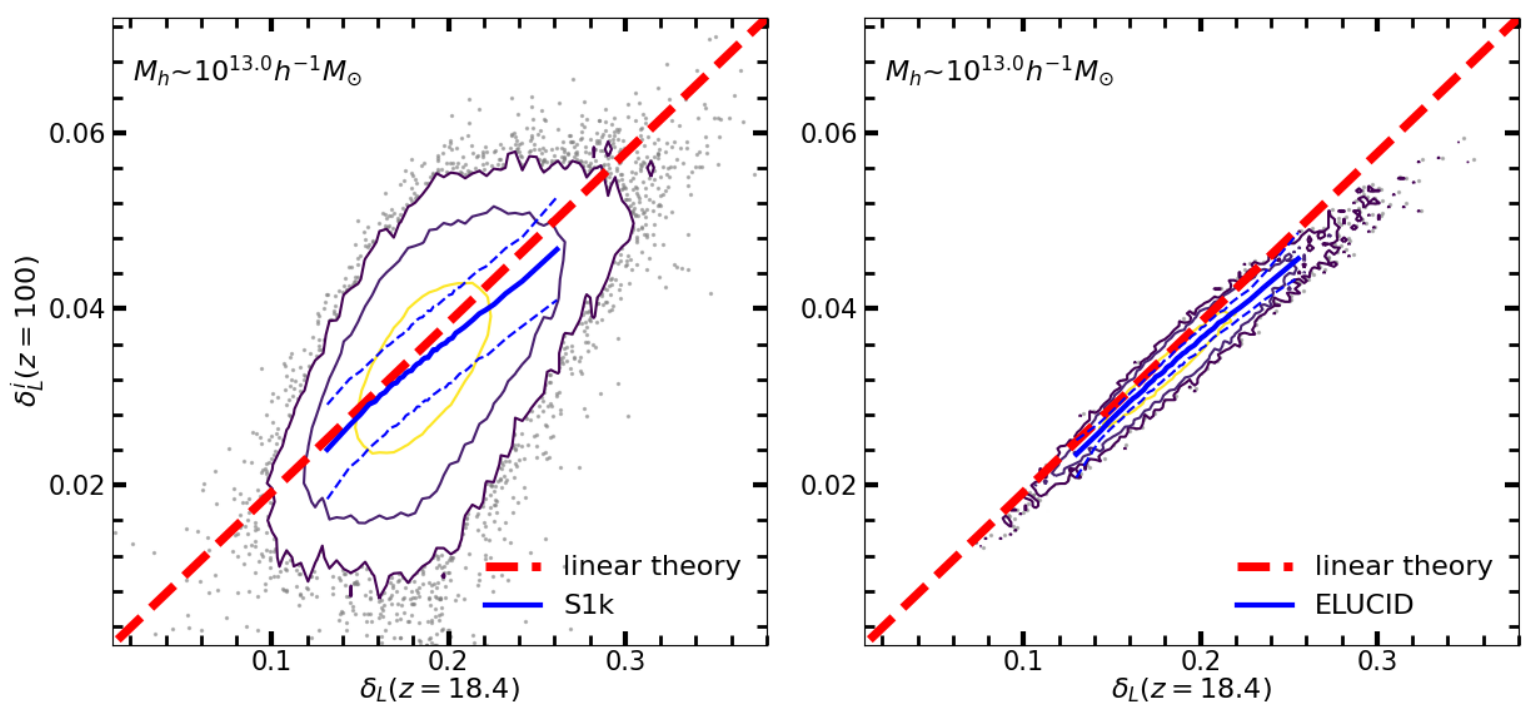

Fig. A.2. Similar to Fig. A.1 but for halos with $\log M_{\mathrm{h}}=13.0$. The left and right panels show the results for S1k and ELUCID, respectively. 